\title{
A discontinuous Sturm-Liouville problem with boundary conditions rationally dependent on the eigenparameter
}

\author{
Zhaowen Zheng ${ }^{1 *}$, Jinming Cai ${ }^{1}$, Kun Li ${ }^{1}$ and Maozhu Zhang ${ }^{2}$
}

\section{"Correspondence:}

zhwzheng@126.com

'School of Mathematical Sciences,

Qufu Normal University, Qufu,

P.R. China

Full list of author information is

available at the end of the article

\begin{abstract}
The present paper deals with a class of discontinuous Sturm-Liouville problems with boundary conditions rationally dependent on the eigenparameter. Operator formulation is built and asymptotic formulas for eigenvalues and eigenfunctions are given. Moreover, the completeness of its eigenfunctions is also discussed.
\end{abstract}

Keywords: Sturm-Liouville problem; Eigenparameter dependent boundary condition; Transmission condition; Asymptotic formulae of eigenvalue and eigenfunction; Completeness

\section{Introduction}

It is well known that the Sturm-Liouville problem with eigenparameter dependent boundary conditions is of great importance for its wide applications in mechanics, mathematical physics, and engineering (see [1-4] and the corresponding references cited therein). It happens at any time by using the approach of separation of variables to solve suitable partial differential equations with boundary conditions containing a directional derivative. Particularly, some examples of spectral problems appearing in mechanical engineering and containing eigenparameter on the boundary conditions were listed in the classical book [1], and Friedman in [5] investigated the method of operator formula to solve the Sturm-Liouville problems with eigenparameter dependent boundary conditions. Later, a large amount of such problems have been considered (see [6-30] and the references cited therein). Particularly, Walter in [4] considered Sturm-Liouville problem with boundary condition dependent on the eigenparameter. They obtained the asymptotic formulas of eigenvalues and eigenfunctions, the expansion theorem was also involved. In [21], Binding et al. considered the Sturm-Liouville problem with the right boundary condition rationally dependent on the eigenparameter and got the existence of eigenvalues and the oscillation theory of eigenfunctions. The authors in [22] also discussed the inverse problems in terms of given spectral data.

Meanwhile, lots of researchers are interested in the discontinuous Sturm-Liouville problems connected with discontinuous material properties, such as heat and mass transfer (see [31]), vibrating string problems when the string loaded additionally with point masses, and diffraction problems. To deal with interior discontinuities, some conditions,

ind changes were made. 
which are often called transmission conditions [10-13], interface conditions [14, 16], are imposed on the discontinuous points. Mukhtarov et al. in [13] considered eigenparameter dependent Sturm-Liouville problems with transmission conditions and obtained some properties of eigenvalues. Also, a two interior discontinuous points case was investigated in $[18,20]$. Numerous authors have been devoted to this area (see [10-20]). By using Mukhtarov's method (see, for example, [10, 12, 13, 20]), the authors defined a new inner product in the direct sum of classical Hilbert spaces in such a way that the considered problem can be interpreted as an eigenvalue problem for a self-adjoint operator with compact resolvent. Most of authors in these literature works focus on the asymptotic formula of eigenvalues and eigenfunctions, oscillation theory, etc. Moreover, what calls for special attention is that the eigenparameter dependent boundary conditions are of rational fraction of parameter $\lambda$ of order one except [21, 22]. Inspired by the above-mentioned literature, we consider the following discontinuous Sturm-Liouville equation:

$$
L y:=-y^{\prime \prime}(x)+q(x) y(x)=\lambda y(x), \quad x \in[0, \xi) \cup(\xi, 1], \xi \in(0,1)
$$

subject to the boundary conditions

$$
\begin{aligned}
& L_{1} y:=\frac{y^{\prime}(0)}{y(0)}=f(\lambda), \\
& L_{2} y:=\frac{y^{\prime}(1)}{y(1)}=g(\lambda),
\end{aligned}
$$

and the transmission conditions

$$
\begin{aligned}
& L_{3} y:=y(\xi+0)-\delta_{1} y(\xi-0)=0, \\
& L_{4} y:=y^{\prime}(\xi+0)-\delta_{2} y^{\prime}(\xi-0)=0,
\end{aligned}
$$

where $\lambda \in \mathbb{C}$ is a spectral parameter; $q(x)$ is a real-valued continuous function on $[0, \xi) \cup$ $(\xi, 1]$ with finite limits $q(\xi \pm 0)=\lim _{x \rightarrow(\xi \pm 0)} q(x) ; \delta_{1}$ and $\delta_{2}$ are nonzero real numbers and $\delta_{1} \delta_{2}>0 ; f(\lambda)$ and $g(\lambda)$ are rational functions of the form

$$
f(\lambda)=-a \lambda+b+\sum_{i=1}^{M} \frac{b_{i}}{\lambda-c_{i}},
$$

where all the coefficients are real and $a \geq 0, b_{i}>0(i=1,2, \ldots, M), c_{1} \leq c_{2} \leq \cdots \leq c_{M}, M$ is finite and is allowed to be 0 (in this case, there are no $c_{i}$ ). If $f(\lambda)=\infty$, then $\frac{y^{\prime}(0)}{y(0)}=f(\lambda)$ is seen as a Dirichlet boundary condition $y(0)=0$.

$$
g(\lambda)=c \lambda+d+\sum_{j=1}^{N} \frac{d_{j}}{e_{j}-\lambda},
$$

where all the coefficients are real and $c \geq 0, d_{j}>0(j=1,2, \ldots, N), e_{1} \leq e_{2} \leq \cdots \leq e_{N}, N$ is finite and is allowed to be 0 (in this case, there are no $e_{j}$ ). If $g(\lambda)=\infty$, then $\frac{y^{\prime}(1)}{y(1)}=g(\lambda)$ is seen as a Dirichlet boundary condition $y(1)=0$. Recall that $f(\lambda)$ and $g(\lambda)$ are sometimes called Herglotz-Nevanlinna type (see [21, 22]). 
In this paper, we research a discontinuous Sturm-Liouville problem with both of the boundary conditions rationally dependent on the eigenparameter $\lambda$. Using classical analysis and operator theoretic formulation, we define a new self-adjoint operator $A$ in a new Hilbert space so that the eigenvalues of such a problem coincide with those of $A$.

The organization of the paper is as follows: after this section, we give the operator formulation in Sect. 2 and get the asymptotic formulas for eigenvalues and eigenfunctions of the problem in Sect. 3. Finally, we discuss the completeness of its eigenfunctions in Sect. 4.

\section{Operator formulation in a new Hilbert space}

In what follows, we always assume that $a>0, c>0$. Firstly, we introduce the Hilbert space $H=L^{2}[0, \xi) \oplus L^{2}(\xi, 1] \oplus \mathbb{C}^{M+N+2}$, the inner product on $H$ is defined by

$$
\begin{aligned}
\langle Y, Z\rangle= & \delta_{1} \delta_{2} \int_{0}^{\xi} y(x) \overline{z(x)} d x+\int_{\xi}^{1} y(x) \overline{z(x)} d x \\
& +\delta_{1} \delta_{2}\left(\sum_{i=1}^{M} \frac{y_{i} \overline{z_{i}}}{b_{i}}+\frac{y_{M+1} \overline{z_{M+1}}}{a}\right)+\sum_{j=1}^{N} \frac{g_{j} \overline{r_{j}}}{d_{j}}+\frac{g_{N+1} \overline{r_{N+1}}}{c}
\end{aligned}
$$

for $Y=\left(y(x), y_{1}, y_{2}, \ldots, y_{M+1}, g_{1}, g_{2}, \ldots, g_{N+1}\right)^{T}, Z=\left(z(x), z_{1}, z_{2}, \ldots, z_{M+1}, r_{1}, r_{2}, \ldots, r_{N+1}\right)^{T} \in$ $H$, and $y(x), z(x) \in L^{2}[0, \xi) \oplus L^{2}(\xi, 1]$. For the convenience, note $H_{1}=L^{2}[0, \xi) \oplus L^{2}(\xi, 1]$.

In the Hilbert space $H$, we consider the operator $A$ as follows:

$$
\begin{aligned}
D(A)= & \left\{Y=\left(y(x), T_{0}(y), T_{1}(y)\right)^{T} \in H \mid y(x), y^{\prime}(x)\right. \text { are absolutely continuous } \\
& \text { on }[0, \xi) \cup(\xi, 1] \text { with finite limits } y(\xi \pm 0), y^{\prime}(\xi \pm 0) \text { and satisfy } \\
& L y \in L^{2}[0, \xi) \oplus L^{2}(\xi, 1], T_{0}(y)=\left(y_{1}, y_{2}, \ldots, y_{M+1}\right), T_{1}(y)=\left(g_{1}, g_{2}, \ldots, g_{N+1}\right), \\
& y_{M+1}=-a y(0), g_{N+1}=c y(1), y(\xi+0)=\delta_{1} y(\xi-0), \\
& \left.y^{\prime}(\xi+0)=\delta_{2} y^{\prime}(\xi-0), y(x) \in H_{1}\right\},
\end{aligned}
$$

which acts by the rule

$$
A Y=\left(\begin{array}{c}
-y^{\prime \prime}(x)+q(x) y(x) \\
c_{1} y_{1}+b_{1} y(0) \\
\vdots \\
c_{M} y_{M}+b_{M} y(0) \\
y^{\prime}(0)-b y(0)-\sum_{i=1}^{M} y_{i} \\
e_{1} g_{1}-d_{1} y(1) \\
\vdots \\
e_{N} g_{N}-d_{N} y(1) \\
y^{\prime}(1)-d y(1)-\sum_{j=1}^{N} g_{j}
\end{array}\right) \text { with } Y=\left(\begin{array}{c}
y(x) \\
y_{1} \\
\vdots \\
y_{M} \\
y_{M+1} \\
g_{1} \\
\vdots \\
g_{N} \\
g_{N+1}
\end{array}\right) \in D(A) .
$$

Then Sturm-Liouville problem (1.1)-(1.5) can be rewritten in the operator-theoretic formulation as $A Y=\lambda Y$ for $Y=\left(y(x), y_{1}, \ldots y_{M}, y_{M+1}, g_{1}, g_{2}, \ldots, g_{N+1}\right)^{T} \in D(A)$. Obviously, the following lemmas hold. 
Lemma 2.1 The eigenvalues and eigenfunctions of Sturm-Liouville problem (1.1)-(1.5) correspond to the eigenvalues and the first element of the corresponding eigenfunctions of operator A, respectively.

Lemma 2.2 The domain $D(A)$ is dense in $H$.

Definition 2.1 Set $W(f, g ; x)=f(x) g^{\prime}(x)-f^{\prime}(x) g(x)$ expresses the Wronskians of functions $f(x)$ and $g(x)$.

Theorem 2.1 Operator $A$ is a symmetric operator in $H$.

Proof For every $Y, Z \in D(A)$, by the definition of inner product and operator $A$ in the new Hilbert space $H$ and integration by parts, we obtain

$$
\begin{aligned}
\langle A Y, Z\rangle-\langle Y, A Z\rangle= & \delta_{1} \delta_{2} W(\bar{z}, y ; 0)-\delta_{1} \delta_{2} W(\bar{z}, y ; \xi-0)-W(\bar{z}, y ; 1) \\
& +W(\bar{z}, y ; \xi+0)-\delta_{1} \delta_{2} W(\bar{z}, y ; 0)+W(\bar{z}, y ; 1) .
\end{aligned}
$$

By transmission conditions (1.4)-(1.5), we get

$$
W(\bar{z}, y ; \xi+0)=\delta_{1} \delta_{2} W(\bar{z}, y ; \xi-0)
$$

Substituting (2.2) into (2.1) yields that $\langle A Y, Z\rangle-\langle Y, A Z\rangle=0$. Our claim is established.

Further, taking into account Lemma 2.2 and Theorem 2.1, we have the following theorem.

Theorem 2.2 Operator A is self-adjoint.

Proof In order to identify that $A$ is a self-adjoint operator, we should prove for every

$$
Y=\left(y(x), y_{1}, \ldots, y_{M}, y_{M+1}, g_{1}, g_{2}, \ldots, g_{N+1}\right)^{T} \in D(A),
$$

$\langle A Y, W\rangle=\langle Y, T\rangle$ for some $T \in H$ implies that $W \in D(A)$ and $A W=T$, where

$$
\begin{aligned}
& W=\left(w(x), w_{1}, \ldots, w_{M}, w_{M+1}, l_{1}, \ldots, l_{N}, L_{N+1}\right)^{T}, \\
& T=\left(t(x), t_{1}, \ldots, t_{M}, t_{M+1}, s_{1}, \ldots, s_{N}, s_{N+1}\right)^{T} .
\end{aligned}
$$

Precisely, we need to prove that the following properties hold:

(i) $w(x), w^{\prime}(x)$ are absolutely continuous on $[0, \xi) \cup(\xi, 1], L w \in L^{2}([0, \xi) \cup(\xi, 1])$;

(ii) $t(x)=L w(x)$;

(iii) $L_{3} w=L_{4} w=0$;

(iv) $w_{M+1}=-a w(0), l_{N+1}=c w(1)$;

(v) $t_{k}=c_{k} w_{k}+b_{k} w(0), k=1,2, \ldots, M$;

(vi) $s_{n}=e_{n} l_{n}-d_{n} w(1), n=1,2, \ldots, N$;

(vii) $t_{M+1}=w^{\prime}(0)-b w(0)-\sum_{k=1}^{M} w_{k}$;

(viii) $s_{N+1}=w^{\prime}(1)-d w(1)-\sum_{n=1}^{N} l_{n}$. 
For an arbitrary point $Y \in C_{0}^{\infty} \oplus 0 \subset D(A)$ (where $0 \subset \mathbb{C}^{M+N+2}$ ), we have

$$
\langle L y, w\rangle_{H_{1}}=\langle y, t\rangle_{H_{1}}
$$

namely

$$
\delta_{1} \delta_{2} \int_{0}^{\xi}(L y(x)) \overline{w(x)} d x+\int_{\xi}^{1}(L y(x)) \overline{w(x)} d x=\delta_{1} \delta_{2} \int_{0}^{\xi} y(x) \overline{t(x)} d x+\int_{\xi}^{1} y(x) \overline{t(x)} d x
$$

In accordance with the classical Sturm-Liouville theory, (i) and (ii) hold. So $\langle A Y$, $W\rangle=$ $\langle Y, T\rangle, \forall Y \in D(A)$ implies

$$
\begin{aligned}
& \langle L y, w\rangle_{H_{1}}+\delta_{1} \delta_{2} \sum_{k=1}^{M} \frac{\left[c_{k} y_{k}+b_{k} y(0)\right] \overline{w_{k}}}{b_{k}}+\frac{\left[y^{\prime}(0)-b y(0)-\sum_{k=1}^{M} y_{k}\right] \overline{w_{M+1}}}{a} \\
& \quad+\sum_{n=1}^{N} \frac{\left[e_{n} g_{n}-d_{n} y(1)\right] \overline{l_{n}}}{d_{n}}+\frac{\left[y^{\prime}(1)-d y(1)-\sum_{n=1}^{N} g_{n}\right] \overline{l_{N+1}}}{c} \\
& =\langle y, L w\rangle_{H_{1}}+\delta_{1} \delta_{2}\left(\sum_{k=1}^{M} \frac{y_{k} \overline{t_{k}}}{b_{k}}+\frac{y_{M+1} \overline{t_{M+1}}}{a}\right)+\sum_{n=1}^{N} \frac{g_{n} \overline{s_{n}}}{d_{n}}+\frac{g_{N+1} \overline{s_{N+1}}}{c} .
\end{aligned}
$$

Besides, by integration by parts we get

$$
\begin{aligned}
\langle L y, w\rangle_{H_{1}}-\langle y, L w\rangle_{H_{1}}= & \delta_{1} \delta_{2}[W(y, \bar{w} ; \xi-0)-W(y, \bar{w} ; 0)] \\
& +W(y, \bar{w} ; 1)-W(y, \bar{w} ; \xi+0) .
\end{aligned}
$$

Combining (2.4) with (2.3), we have

$$
\begin{aligned}
\delta_{1} \delta_{2}[ & \left.\sum_{k=1}^{M} \frac{y_{k} \overline{t_{k}}}{b_{k}}-y(0) \overline{t_{M+1}}\right]+\sum_{n=1}^{N} \frac{g_{n} \overline{s_{n}}}{d_{n}}+y(1) \overline{s_{N+1}} \\
& -\delta_{1} \delta_{2} \sum_{k=1}^{M} \frac{\left[c_{k} y_{k}+b_{k} y(0)\right] \overline{w_{k}}}{b_{k}}+\frac{\left[y^{\prime}(0)-b y(0)-\sum_{k=1}^{M} y_{k}\right] \overline{w_{M+1}}}{a} \\
& -\sum_{n=1}^{N} \frac{\left[e_{n} g_{n}-d_{n} y(1)\right] \overline{l_{n}}}{d_{n}}-\frac{\left[y^{\prime}(1)-d y(1)-\sum_{n=1}^{N} g_{n}\right] \overline{l_{N+1}}}{c} \\
= & \delta_{1} \delta_{2}[W(y, \bar{w} ; \xi-0)-W(y, \bar{w} ; 0)]+W(y, \bar{w} ; 1)-W(y, \bar{w} ; \xi+0) .
\end{aligned}
$$

By Naimark's patching lemma [24], there exists $Y \in D(A)$ such that

$$
\begin{aligned}
& y(0)=y(1)=y^{\prime}(1)=y(\xi \pm 0)=y^{\prime}(\xi \pm 0)=0 \neq y^{\prime}(0), \\
& y_{k}=0 \quad(k=1,2, \ldots, M), \quad g_{n}=0 \quad(n=1,2, \ldots, N) .
\end{aligned}
$$

Substituting them into (2.5), we obtain $w_{M+1}=-a w(0)$. Similarly, we can find $Y \in D(A)$ such that

$$
y(0)=y^{\prime}(0)=y(1)=y(\xi \pm 0)=y^{\prime}(\xi \pm 0)=0 \neq y^{\prime}(1)
$$




$$
y_{k}=0 \quad(k=1,2, \ldots, M), \quad g_{n}=0 \quad(n=1,2, \ldots, N) .
$$

We can get $l_{N+1}=c w(1)$. So (iv) holds. Next, set $Y \in D(A)$ such that

$$
\begin{aligned}
& y(0)=y^{\prime}(0)=y(1)=y^{\prime}(1)=y(\xi \pm 0)=0, \\
& y_{k}=0 \quad(k=1,2, \ldots, M), \quad g_{n}=0 \quad(n=1,2, \ldots, N), \\
& y^{\prime}(\xi-0)=\delta_{1}, \quad y^{\prime}(\xi+0)=\delta_{1} \delta_{2} .
\end{aligned}
$$

Then, by (2.5), we get $L_{3} w=0$. Analogously, we can get $L_{4} w=0$.

When we choose $Y \in D(A)$ which satisfies

$$
\begin{aligned}
& y(0)=y^{\prime}(0)=y(1)=y^{\prime}(1)=y(\xi \pm 0)=y^{\prime}(\xi \pm 0)=0, \\
& g_{n}=0 \quad(n=1,2, \ldots, N), \quad y_{k}=\delta_{i k}
\end{aligned}
$$

(v) holds.

When we choose $Y \in D(A)$ which satisfies

$$
\begin{aligned}
& y(0)=y^{\prime}(0)=y(1)=y^{\prime}(1)=y(\xi \pm 0)=y^{\prime}(\xi \pm 0)=0, \\
& y_{k}=0 \quad(k=1,2, \ldots, M), \quad g_{n}=\delta_{j n},
\end{aligned}
$$

(vi) holds.

When we choose $Y \in D(A)$ which satisfies

$$
\begin{aligned}
& y^{\prime}(0)=y(1)=y^{\prime}(1)=y(\xi \pm 0)=y^{\prime}(\xi \pm 0)=0 \neq y(0), \\
& y_{k}=0 \quad(k=1,2, \ldots, M), \quad g_{n}=0 \quad(n=1,2, \ldots, N),
\end{aligned}
$$

(vii) holds. Similarly, we have (viii). So the operator $A$ is a self-adjoint operator.

From the characteristics of self-adjoint operators, we have the following.

Corollary 2.1 All eigenvalues of Sturm-Liouville problem (1.1)-(1.5) are real.

Corollary 2.2 Let $\lambda_{1}$ and $\lambda_{2}$ be two different eigenvalues of Sturm-Liouville problem (1.1)(1.5), then the corresponding eigenfunctions $y(x)$ and $z(x)$ are orthogonal in the sense of

$$
\begin{aligned}
& \delta_{1} \delta_{2} \int_{0}^{\xi} y(x) \overline{z(x)} d x+\int_{\xi}^{1} y(x) \overline{z(x)} d x \\
& \quad+\delta_{1} \delta_{2}\left(\sum_{i=1}^{M} \frac{y_{i} \overline{z_{i}}}{b_{i}}+\frac{y_{M+1} \overline{z_{M+1}}}{a}\right)+\sum_{j=1}^{N} \frac{g_{j} \overline{r_{j}}}{d_{j}}+\frac{g_{N+1} \overline{r_{N+1}}}{c}=0 .
\end{aligned}
$$

\section{Asymptotic formulas for eigenvalues and eigenfunctions}

We rebuild the fundamental solutions of Sturm-Liouville problem (1.1)-(1.5) and get the asymptotic formulas for the eigenvalues and eigenfunctions in this section. 
Lemma 3.1 (see [26]) Let the real-valued function $q(x)$ be continuous on $I=[0, \xi) \cup(\xi, 1]$, and $f(\lambda), g(\lambda)$ be given entire functions. Then, for any $\lambda \in \mathbb{C}$, the following equation

$$
L y:=-y^{\prime \prime}(x)+q(x) y(x)=\lambda y(x), \quad x \in[0, \xi) \cup(\xi, 1]
$$

has the unique solution $y=y(x, \lambda)$ satisfying the initial conditions

$$
y(0, \lambda)=f(\lambda), \quad y^{\prime}(0, \lambda)=g(\lambda) .
$$

For each fixed $x \in[0, \xi) \cup(\xi, 1], y(x, \lambda)$ is an entire function of $\lambda$.

Now, we define fundamental solutions $\varphi(x, \lambda)$ and $\chi(x, \lambda)$ of equation (1.1) by the following procedure, where

$$
\varphi(x, \lambda)= \begin{cases}\varphi_{1}(x, \lambda), & x \in[0, \xi), \\ \varphi_{2}(x, \lambda), & x \in(\xi, 1]\end{cases}
$$

and

$$
\chi(x, \lambda)= \begin{cases}\chi_{1}(x, \lambda), & x \in[0, \xi), \\ \chi_{2}(x, \lambda), & x \in(\xi, 1] .\end{cases}
$$

Set $\varphi_{1}(x, \lambda)$ is the solution of equation (1.1) on the interval $[0, \xi)$, which satisfies the initial conditions

$$
\begin{aligned}
& \varphi_{1}(0, \lambda)=1, \\
& \varphi_{1}^{\prime}(0, \lambda)=f(\lambda) .
\end{aligned}
$$

In accordance with Lemma 3.1, we can define the solution $\varphi_{2}(x, \lambda)$ of equation (1.1) on $(\xi, 1]$ by the initial conditions

$$
\left(\begin{array}{l}
\varphi_{2}(\xi+0) \\
\varphi_{2}^{\prime}(\xi+0)
\end{array}\right)=\left(\begin{array}{l}
\delta_{1} \varphi_{1}(\xi-0) \\
\delta_{2} \varphi_{1}^{\prime}(\xi-0)
\end{array}\right)
$$

similarly, we define the solutions $\chi_{2}(x, \lambda)$ and $\chi_{1}(x, \lambda)$ of equation (1.1) by the initial conditions, respectively,

$$
\begin{aligned}
& \chi_{2}(1, \lambda)=1, \\
& \chi_{2}^{\prime}(1, \lambda)=g(\lambda), \\
& \left(\begin{array}{l}
\chi_{1}(\xi-0, \lambda) \\
\chi_{1}^{\prime}(\xi-0, \lambda)
\end{array}\right)=\left(\begin{array}{c}
\frac{\chi_{2}(\xi+0, \lambda)}{\delta_{1}} \\
\frac{\chi_{2}^{\prime}(\xi+0, \lambda)}{\delta_{2}}
\end{array}\right) .
\end{aligned}
$$

Now we consider Wronskians

$$
W_{i}(\lambda):=W\left(\varphi_{i}, \chi_{i} ; x\right)=\varphi_{i}(x, \lambda) \chi_{i}^{\prime}(x, \lambda)-\varphi_{i}^{\prime}(x, \lambda) \chi_{i}(x, \lambda) \quad(i=1,2) .
$$


By the dependence of solutions of initial value problems on the parameter, we have $W_{i}(\lambda)$ $(i=1,2)$ are independent of $x$.

Lemma 3.2 For every $\lambda \in \mathbb{C}, W_{2}(\lambda)=\delta_{1} \delta_{2} W_{1}(\lambda)$.

Proof By the definition of $W_{i}(\lambda)$, we get

$$
\begin{aligned}
& W_{1}(\lambda)=\varphi_{1}(\xi-0, \lambda) \chi_{1}^{\prime}(\xi-0, \lambda)-\varphi_{1}^{\prime}(\xi-0, \lambda) \chi_{1}(\xi-0, \lambda), \\
& W_{2}(\lambda)=\varphi_{2}(\xi+0, \lambda) \chi_{2}^{\prime}(\xi+0, \lambda)-\varphi_{2}^{\prime}(\xi+0, \lambda) \chi_{2}(\xi+0, \lambda) .
\end{aligned}
$$

Using the transmission conditions (1.4)-(1.5), short calculation gives that

$$
W\left(\varphi_{2}, \chi_{2} ; \xi+0\right)=\delta_{1} \delta_{2} W\left(\varphi_{1}, \chi_{1} ; \xi-0\right) .
$$

Thus, for every $\lambda \in \mathbb{C}$, we have $W_{2}(\lambda)=\delta_{1} \delta_{2} W_{1}(\lambda)$, this completes the proof.

Besides, we set $W(\lambda):=W_{1}(\lambda)=\frac{1}{\delta_{1} \delta_{2}} W_{2}(\lambda)$.

Theorem 3.1 The eigenvalues of Sturm-Liouville problem (1.1)-(1.5) coincide with the roots of $W(\lambda)=0$.

Proof Let $v_{0}\left(x, \lambda_{0}\right)$ be any eigenfunction corresponding to eigenvalue $\lambda_{0}$, then the function $v_{0}\left(x, \lambda_{0}\right)$ can be written as the form

$$
v_{0}\left(x, \lambda_{0}\right)= \begin{cases}m_{1} \varphi_{1}\left(x, \lambda_{0}\right)+m_{2} \chi_{1}\left(x, \lambda_{0}\right), & x \in[0, \xi), \\ m_{3} \varphi_{2}\left(x, \lambda_{0}\right)+m_{4} \chi_{2}\left(x, \lambda_{0}\right), & x \in(\xi, 1],\end{cases}
$$

where at least one of the constants $m_{i}(i=1,2,3,4)$ is nonzero. We should show that $W\left(\lambda_{0}\right)=0$. Suppose to the contrary that there exists $\lambda_{0} \in \mathbb{R}$ such that $W\left(\lambda_{0}\right)=W_{1}\left(\lambda_{0}\right)=$ $\frac{1}{\delta_{1} \delta_{2}} W_{2}\left(\lambda_{0}\right) \neq 0$. Since the eigenfunction $v_{0}\left(x, \lambda_{0}\right)$ satisfies both boundary and transmission conditions (1.2)-(1.5), we have $L_{i} v_{0}\left(x, \lambda_{0}\right)=0(i=1,2,3,4)$. However, the determinant of coefficient matrix is nonzero, so we get $m_{i}=0(i=1,2,3,4)$, which is a contradiction. Then $W\left(\lambda_{0}\right)=0$. Conversely, set $W\left(\lambda_{0}\right)=0$, then $W\left(\lambda_{0}\right)=W_{1}\left(\lambda_{0}\right)=\frac{1}{\delta_{1} \delta_{2}} W_{2}\left(\lambda_{0}\right)=0$, therefore, $\chi_{i}\left(x, \lambda_{0}\right)=k \varphi_{i}\left(x, \lambda_{0}\right)(i=1,2)$ for some $k \neq 0$. Since both $\varphi_{2}\left(x, \lambda_{0}\right)$ and $\chi_{2}\left(x, \lambda_{0}\right)$ satisfy the boundary condition (1.3), thus

$$
\varphi\left(x, \lambda_{0}\right)= \begin{cases}\varphi_{1}\left(x, \lambda_{0}\right), & x \in[0, \xi), \\ \varphi_{2}\left(x, \lambda_{0}\right), & x \in[\xi, 1]\end{cases}
$$

satisfies problem (1.1)-(1.5). So the function $\varphi\left(x, \lambda_{0}\right)$ is an eigenfunction of problem (1.1)(1.5) corresponding to eigenvalue $\lambda_{0}$. Our claim is established.

Remark 3.1 We define $y(x, \lambda)$ to be a non-trivial solution of (1.1), (1.4)-(1.5), write $F(\lambda)=$ $y^{\prime}(0, \lambda)-f(\lambda) y(0, \lambda), G(\lambda)=y^{\prime}(1, \lambda)-g(\lambda) y(1, \lambda), W(\lambda)=F(\lambda) G(\lambda)$. If $W(\lambda)=0$, then $\lambda$ is an eigenvalue of (1.1)-(1.5). If, in addition, $W_{\lambda}(\lambda) \neq 0$, then we call $\lambda$ a simple eigenvalue, where the suffix denotes differentiation with respect to $\lambda$. To discuss the poles of $f(\lambda)$, 
we use $\Omega(\lambda)=\left(y(0, \lambda)-\frac{y^{\prime}(0, \lambda)}{f(\lambda)}\right)\left(y(1, \lambda)-\frac{y^{\prime}(1, \lambda)}{g(\lambda)}\right)$ instead of $W(\lambda)$. Particularly, if $y(0, \lambda)=0$, then $\lambda=c_{i}$ is an eigenvalue. In addition, if $y_{\lambda}(0, \lambda) \neq 0$, then $\lambda=c_{i}$ is a simple eigenvalue. If $y(1, \lambda)=0$, then $\lambda=e_{j}$ is an eigenvalue. In addition, if $y_{\lambda}(1, \lambda) \neq 0$, then $\lambda=e_{j}$ is a simple eigenvalue.

Theorem 3.2 All eigenvalues of Sturm-Liouville problem (1.1)-(1.5) are analytically single.

Proof Set $\lambda=s+i$, we use the following notations: $\varphi=\varphi(x, \lambda), \varphi_{1 \lambda}=\frac{\partial \varphi_{1}}{\partial \lambda}, \varphi_{1 \lambda}^{\prime}=\frac{\partial \varphi_{1}^{\prime}}{\partial \lambda}$. Differentiating the equation $A \chi=\lambda \chi$ with respect to $\lambda$, we obtain

$$
A \chi_{\lambda}=\chi+\lambda \chi_{\lambda}
$$

Then

$$
\left\langle\lambda \chi_{\lambda}, \varphi\right\rangle_{H_{1}}-\left\langle\chi_{\lambda}, \lambda \varphi\right\rangle_{H_{1}}=\langle\chi, \varphi\rangle_{H_{1}} .
$$

Using integration by parts, we have

$$
\left\langle A \chi_{\lambda}, \varphi\right\rangle_{1}-\left\langle\chi_{\lambda}, A \varphi\right\rangle_{1}=\left.\delta_{1} \delta_{2}\left(\chi_{1 \lambda}{\overline{\varphi_{1}}}^{\prime}-\chi_{1 \lambda}^{\prime} \overline{\varphi_{1}}\right)\right|_{0} ^{\xi}+\left.\left(\chi_{2 \lambda}{\overline{\varphi_{2}}}^{\prime}-\chi_{2 \lambda}^{\prime} \overline{\varphi_{2}}\right)\right|_{\xi} ^{1} .
$$

Further, by the initial conditions, we have

$$
\begin{aligned}
& \left.\delta_{1} \delta_{2}\left(\chi_{1 \lambda}{\overline{\varphi_{1}}}^{\prime}-\chi_{1 \lambda}^{\prime} \overline{\bar{\varphi}_{1}}\right)\right|_{0} ^{\xi}+\left.\left(\chi_{2 \lambda} \overline{\bar{\varphi}_{2}^{\prime}}-\chi_{2 \lambda}^{\prime} \overline{\varphi_{2}}\right)\right|_{\xi} ^{1} \\
& \quad=\delta_{1} \delta_{2} \chi_{1 \lambda}^{\prime}(0)-\overline{\varphi_{2}(1)}\left[c+\sum_{j=1}^{N} \frac{d_{j}}{\left(e_{j}-\lambda\right)^{2}}\right]-\delta_{1} \delta_{2} \chi_{1 \lambda}(0)\left(-a \lambda+b+\sum_{i=1}^{M} \frac{b_{i}}{\lambda-c_{i}}\right) .
\end{aligned}
$$

By virtue of the definition of $W(\lambda),(3.1)$, and (3.2), we observe that

$$
\begin{aligned}
W(\lambda) & =\varphi_{1}(0, \lambda) \chi_{1}^{\prime}(0, \lambda)-\varphi_{1}^{\prime}(0, \lambda) \chi_{1}(0, \lambda) \\
& =\chi_{1}^{\prime}(0, \lambda)-\left(-a \lambda+b+\sum_{i=1}^{M} \frac{b_{i}}{\lambda-c_{i}}\right) \chi_{1}(0, \lambda) .
\end{aligned}
$$

Differentiating it we get

$$
W^{\prime}(\lambda)=\chi_{1 \lambda}^{\prime}(0, \lambda)-\chi_{1 \lambda}(0, \lambda)\left(-a \lambda+b+\sum_{i=1}^{M} \frac{b_{i}}{\lambda-c_{i}}\right)+\chi_{1}(0, \lambda)\left[a+\sum_{i=1}^{M} \frac{b_{i}}{\left(\lambda-c_{i}\right)^{2}}\right] .
$$

Next, let $\lambda_{0}$ be an arbitrary root of $W(\lambda)=0$. Then we get $\varphi_{i}\left(x, \lambda_{0}\right)=k \chi_{i}\left(x, \lambda_{0}\right)(i=1,2)$ $(k \neq 0), k \in \mathbb{R}$. Noting that $\lambda_{0} \in \mathbb{R}$, by a short calculation, (3.8) becomes

$$
\begin{aligned}
W^{\prime}\left(\lambda_{0}\right)= & \bar{k} \int_{0}^{\xi}\left|\chi_{1}(x)\right|^{2} d x+\frac{\bar{k}}{\delta_{1} \delta_{2}}\left[\int_{\xi}^{1}\left|\chi_{2}(x)\right|^{2} d x+c+\sum_{j=1}^{N} \frac{d_{j}}{\left(e_{j}-\lambda_{0}\right)^{2}}\right] \\
& +\frac{1}{k}\left[a+\sum_{i=1}^{M} \frac{b_{i}}{\left(\lambda_{0}-c_{i}\right)^{2}}\right] .
\end{aligned}
$$


Since $c>0, a>0, b_{i}>0(i=1,2, \ldots, M), d_{j}>0(j=1,2, \ldots, N), \delta_{1} \delta_{2}>0, k \neq 0$, so we know $W^{\prime}\left(\lambda_{0}\right) \neq 0$. Hence the eigenvalues of Sturm-Liouville problem (1.1)-(1.5) are analytically single.

Lemma 3.3 Let $\lambda=s^{2}, s=\sigma+$ it. Then the following equalities hold for $k=0$ and $k=1$ :

$$
\begin{aligned}
\frac{d^{k}}{d x^{k}} \varphi_{1}(x, \lambda)= & \frac{d^{k}}{d x^{k}} \cos (s x)-\left(-a s+\frac{b}{s}+\sum_{i=1}^{M} \frac{b_{i}}{s^{3}-c_{i} s}\right) \frac{d^{k}}{d x^{k}} \sin (s x) \\
& +\frac{1}{s} \int_{0}^{x} \frac{d^{k}}{d x^{k}} \sin [s(x-\tau)] q(\tau) \varphi_{1}(\tau) d \tau, \\
\frac{d^{k}}{d x^{k}} \varphi_{2}(x, \lambda)= & \delta_{1} \varphi_{1}(\xi-0) \frac{d^{k}}{d x^{k}} \cos [s(x-\xi)]+\frac{1}{s} \delta_{2} \varphi_{1}^{\prime}(\xi-0) \frac{d^{k}}{d x^{k}} \sin [s(x-\xi)] \\
& +\frac{1}{s} \int_{\xi}^{x} \frac{d^{k}}{d x^{k}} \sin [s(x-\tau)] q(\tau) \varphi_{2}(\tau) d \tau .
\end{aligned}
$$

Proof For the case of $k=0$, since $-\varphi_{1}^{\prime \prime}+q \varphi_{1}=s^{2} \varphi_{1}$, we have $q \varphi_{1}=\varphi_{1}^{\prime \prime}+s^{2} \varphi_{1}$, and

$$
\int_{0}^{x} \sin [s(x-\tau)] q(\tau) \varphi_{1}(\tau) d \tau=s^{2} \int_{0}^{x} \varphi_{1}(\tau) \sin [s(x-\tau)] d \tau+\int_{0}^{x} \sin [s(x-\tau)] \varphi_{1}^{\prime \prime}(\tau) d \tau
$$

Using integration by parts and noting the initial conditions

$$
\varphi_{1}(0)=1, \quad \varphi_{1}^{\prime}(0)=-a s^{2}+b+\sum_{i=1}^{M} \frac{b_{i}}{s^{2}-c_{i}}
$$

we obtain

$$
s \varphi_{1}(x, \lambda)=\left(-a s^{2}+b+\sum_{i=1}^{M} \frac{b_{i}}{s^{2}-c_{i}}\right) \sin (s x)+s \cos (s x)+\int_{0}^{x} \sin [s(x-\tau)] q(\tau) \varphi_{1}(\tau) d \tau .
$$

Thus,

$$
\begin{aligned}
\varphi_{1}(x, \lambda)= & \left(-a s+\frac{b}{s}+\sum_{i=1}^{M} \frac{b_{i}}{s^{3}-c_{i} s}\right) \sin (s x)+\cos (s x) \\
& +\frac{1}{s} \int_{0}^{x} \sin [s(x-\tau)] q(\tau) \varphi_{1}(\tau) d \tau .
\end{aligned}
$$

Then (3.9) can be got by differentiating (3.11) with respect to $x$. The proof for (3.10) is similar.

Similarly, we have the following lemma.

Lemma 3.4 Let $\lambda=s^{2}, s=\sigma+i$. Then the following equalities hold for $k=0$ and $k=1$ :

$$
\begin{aligned}
\frac{d^{k}}{d x^{k}} \chi_{1}(x, \lambda)= & \frac{\chi_{2}(\xi+0)}{\delta_{1}} \frac{d^{k}}{d x^{k}} \cos [s(x-\xi)]+\frac{\chi_{2}^{\prime}(\xi+0)}{s \delta_{2}} \frac{d^{k}}{d x^{k}} \sin [s(x-\xi)] \\
& -\frac{1}{s} \int_{x}^{\xi} \frac{d^{k}}{d x^{k}} \sin [s(x-\tau)] q(\tau) \chi_{1}(\tau) d \tau
\end{aligned}
$$




$$
\begin{aligned}
\frac{d^{k}}{d x^{k}} \chi_{2}(x, \lambda)= & \frac{d^{k}}{d x^{k}} \cos [s(x-1)]+\left(c s+\frac{d}{s}-\sum_{j=1}^{N} \frac{d_{j}}{e_{j} s-s^{3}}\right) \frac{d^{k}}{d x^{k}} \sin [s(x-1)] \\
& -\frac{1}{s} \int_{x}^{1} \frac{d^{k}}{d x^{k}} \sin [s(x-\tau)] q(\tau) \chi_{2}(\tau) d \tau
\end{aligned}
$$

Lemma 3.5 Let $\lambda=s^{2}, s=\sigma+i t$. Then $\varphi(x, \lambda)$ have the following asymptotic representations for $k=0,1$ :

$$
\begin{aligned}
\frac{d^{k}}{d x^{k}} \varphi_{1}(x, \lambda)= & -s a \frac{d^{k}}{d x^{k}} \sin (s x)+O\left(|s|^{k} e^{|t| x}\right), \\
\frac{d^{k}}{d x^{k}} \varphi_{2}(x, \lambda)= & -s a \sin (s \xi) \frac{d^{k}}{d x^{k}} \cos [s(x-\xi)] \\
& -s a \delta_{2} \cos (s \xi) \frac{d^{k}}{d x^{k}} \sin [s(x-\xi)]+O\left(|s|^{k} e^{|t| x}\right) .
\end{aligned}
$$

Each of these estimations holds uniformly for $x$ as $|\lambda| \rightarrow \infty$.

Proof The proof of asymptotic equalities for $\varphi_{1}(x, \lambda)$ are similar to those of Titchmarsh's proof for $\varphi(x, \lambda)$ (see [25]), so we only prove (3.14), the other asymptotic equalities are similar.

For the case of $k=0$, by the estimations of $\varphi_{1}(x, \lambda)$ and $\varphi_{1}^{\prime}(x, \lambda)$, we get

$$
\begin{aligned}
& \varphi_{1}(\xi-0, \lambda)=-s a \sin (s \xi)+O\left(|s| e^{|t| \xi}\right), \\
& \varphi_{1}^{\prime}(\xi-0, \lambda)=-s^{2} a \cos (s \xi)+O\left(|s|^{2} e^{|t| \xi}\right) .
\end{aligned}
$$

Substituting them into (3.10) and noting (3.3), we have

$$
\varphi_{2}(x, \lambda)=-s a \sin (s \xi) \cos [s(x-\xi)]-s a \delta_{2} \cos (s \xi) \sin [s(x-\xi)]+O\left(|s| e^{|t| x}\right)
$$

Then, differentiating it with respect to $x$, we obtain (3.14).

Lemma 3.6 Let $\lambda=s^{2}, s=\sigma+i$. Then $\chi(x, \lambda)$ have the following asymptotic representations for $k=0,1$ :

$$
\begin{aligned}
\frac{d^{k}}{d x^{k}} \chi_{1}(x, \lambda)= & -\frac{s c}{\delta_{1}} \sin [s(\xi-1)] \frac{d^{k}}{d x^{k}} \cos [s(x-\xi)] \\
& +\frac{s c}{\delta_{2}} \cos [s(\xi-1)] \frac{d^{k}}{d x^{k}} \sin [s(x-\xi)]+O\left(|s|^{k} e^{|t|(x-1)}\right), \\
\frac{d^{k}}{d x^{k}} \chi_{2}(x, \lambda)= & s c \frac{d^{k}}{d x^{k}} \sin [s(x-1)]+O\left(|s|^{k} e^{|t|(x-1)}\right) .
\end{aligned}
$$

Each of these estimations holds uniformly for $x$ as $|\lambda| \rightarrow \infty$.

Theorem 3.3 Let $\lambda=s^{2}, s=\sigma+i$. The function $W_{1}(\lambda)$ has the following estimations:

$$
W_{1}(\lambda)=\frac{a c s^{3} \cos (s \xi) \sin [s(\xi-1)]}{\delta_{1}}-\frac{a c s^{3} \sin (s \xi) \cos [s(\xi-1)]}{\delta_{2}}+O\left(|s|^{2} e^{|t|}\right) .
$$


Proof By the definition of $W_{1}(\lambda)$, we have

$$
\begin{aligned}
W_{1}(\lambda) & =\varphi_{1}(0, \lambda) \chi_{1}^{\prime}(0, \lambda)-\varphi_{1}^{\prime}(0, \lambda) \chi_{1}(0, \lambda) \\
& =\chi_{1}^{\prime}(0, \lambda)-\left(-s a+\frac{b}{s}+\sum_{i=1}^{M} \frac{b_{i}}{s^{3}-c_{i} s}\right) s \chi_{1}(0, \lambda) .
\end{aligned}
$$

According to the estimations of $\chi_{1}(0, \lambda)$ and $\chi_{1}^{\prime}(0, \lambda)$ in Lemma 3.6, we can obtain the asymptotic representations of $W_{1}(\lambda)$.

Corollary 3.1 The eigenvalues of Sturm-Liouville problem (1.1)-(1.5) are bounded below.

Proof Setting $s=i t(t>0)$ in Theorem 3.3, we get $W_{1}(\lambda)=W_{1}\left(-t^{2}\right) \rightarrow \infty(t \rightarrow \infty)$. Then $W_{1}\left(-t^{2}\right) \neq 0$ for $\lambda$ negative and sufficiently large. Our claim is established.

Further, according to the asymptotic representations for $W_{1}(\lambda)$, we have the following theorem. For the convenience, in the sequel, we assume $\delta_{1}=\delta_{2}=\delta$.

Theorem 3.4 The eigenvalues $\lambda_{n}=s_{n}^{2}(n=0,1,2, \ldots)$ of discontinuous Sturm-Liouville problem (1.1)-(1.5) have the following estimations as $n \rightarrow \infty$ :

$$
\sqrt{\lambda_{n}}=(n-1) \pi+O\left(\frac{1}{n}\right)
$$

Proof Using the well-known Rouché theorem in a closed curve removing $c_{i}(i=1,2, \ldots, M)$ and $e_{j}(j=1,2, \ldots, N)$, we can obtain this result (see [23], Theorem 2.3).

Combining Theorem 3.4, Lemma 3.5 with Lemma 3.6, we can obtain the following asymptotic representations of the eigenfunctions $\varphi\left(x, \lambda_{n}\right)$ and $\chi\left(x, \lambda_{n}\right)$ :

Theorem 3.5 The eigenfunctions $\varphi\left(x, \lambda_{n}\right)$ and $\chi\left(x, \lambda_{n}\right)(n=0,1,2, \ldots)$ of Sturm-Liouville problem (1.1)-(1.5) have the following asymptotic representations as $n \rightarrow \infty$ :

$$
\begin{aligned}
& \varphi\left(x, \lambda_{n}\right)= \begin{cases}-a(n-1) \pi \sin [(n-1) \pi x]+O(1), & x \in[0, \xi), \\
-a \delta(n-1) \pi \sin [(n-1) \pi x]+O(1), & x \in(\xi, 1] .\end{cases} \\
& \chi\left(x, \lambda_{n}\right)= \begin{cases}\pi c \frac{(n-1)}{\delta} \sin [(n-1) \pi(x-1)]+O(1), & x \in[0, \xi), \\
c(n-1) \pi \sin [(n-1) \pi(x-1)]+O(1), & x \in(\xi, 1] .\end{cases}
\end{aligned}
$$

\section{Completeness of eigenfunctions}

In this section, we get the property of spectrum for the operator $A$ and discuss the completeness of the eigenfunctions of problem (1.1)-(1.5) .

Theorem 4.1 The operator $A$ has only point spectrum, i.e., $\sigma(A)=\sigma_{p}(A)$.

Proof We claim that if $\theta$ is not an eigenvalue of $A$, then $\theta$ must be in the resolvent set of $A$. The spectrum of $A$ will consist of eigenvalues accumulating only at $\infty$, and this will complete the proof. According to Theorem 2.2, we only need to consider real $\theta$. The $L^{2}$ 
component of $(A-\theta) Y=\widetilde{H} \in H$, where $\theta \in \mathbb{R}$ involves the equations $L y=h$, where $Y=$ $\left(y(x), y_{1}, \ldots y_{M}, y_{M+1}, g_{1}, g_{2}, \ldots, g_{N+1}\right)^{T}, \widetilde{H}=\left(h(x), h_{1}, \ldots h_{M}, h_{M+1}, \varpi_{1}, \varpi_{2}, \ldots, \varpi_{N+1}\right)^{T}$. Consider the problem

$$
\left\{\begin{array}{l}
-y^{\prime \prime}(x)+q(x) y(x)-\theta y(x)=h(x), \quad x \in[0, \xi) \cup(\xi, 1] \\
y(\xi+0)=\delta_{1} y(\xi-0) \\
y^{\prime}(\xi+0)=\delta_{2} y^{\prime}(\xi-0)
\end{array}\right.
$$

Let

$$
\psi(x)= \begin{cases}\psi_{1}(x), & x \in[0, \xi) \\ \psi_{2}(x), & x \in(\xi, 1]\end{cases}
$$

be the solution of the equation $-y^{\prime \prime}(x)+q(x) y(x)-\theta y(x)=0$ satisfying the transmission conditions (1.4)-(1.5). Let

$$
\phi(x)= \begin{cases}\phi_{1}(x), & x \in[0, \xi), \\ \phi_{2}(x), & x \in(\xi, 1]\end{cases}
$$

be the solution of the equation $-y^{\prime \prime}(x)+q(x) y(x)-\theta y(x)=h(x)$ satisfying the transmission conditions (1.4)-(1.5). Then equation (4.1) has the general solution

$$
y(x)= \begin{cases}\mu \psi_{1}(x)+\phi_{1}(x), & x \in[0, \xi), \\ \mu \psi_{2}(x)+\phi_{2}(x), & x \in(\xi, 1],\end{cases}
$$

where $\mu \in \mathbb{C}$.

Observe that

$$
\begin{aligned}
& y^{\prime}(0)-y(0) f(\theta) \neq 0, \\
& y^{\prime}(1)-y(1) g(\theta) \neq 0 .
\end{aligned}
$$

The next $M+1$ components of $(A-\theta) Y=\widetilde{H} \in H$ lead to

$$
\begin{aligned}
& \left(c_{i}-\theta\right) y_{i}=\theta h_{i}-b_{i} y(0) \quad(i=1,2, \ldots, M), \\
& y^{\prime}(0)-b y(0)-\sum_{i=1}^{M} y_{i}+a \theta y(0)=h_{M+1}
\end{aligned}
$$

The next $N+1$ components of $(A-\theta) Y=\widetilde{H} \in H$ lead to

$$
\begin{aligned}
& \left(e_{j}-\theta\right) g_{j}=\theta \varpi_{j}+d_{j} y(1) \quad(j=1,2, \ldots, N), \\
& y^{\prime}(1)-d y(1)-\sum_{j=1}^{N} g_{j}-\theta c y(1)=\varpi_{N+1} .
\end{aligned}
$$


Substituting (4.4) and (4.7) into (4.8), substituting (4.4) and (4.9) into (4.10), we can get that $\mu, g_{j}$ and $y_{i}$ are uniquely solvable. So $y(x)$ is uniquely determined. Noting that $(A-\theta I)^{-1}$ is defined on all of $H$. We obtain that $(A-\theta I)^{-1}$ is bounded by Theorem 2.2 and the closed graph theorem. Thus $\theta \in \rho(A)$. Hence our claim is established.

Lemma 4.1 The operator $A$ has compact resolvent, that is, for each $\delta \in \mathbb{R} / \sigma_{p}(A),(A-\delta I)^{-1}$ is compact on $H$ (see [19], Theorem 6.3.3).

Using the above lemma and the spectral mapping theorem, we also get the following theorem.

Theorem 4.2 The eigenfunctions of Sturm-Liouville problem (1.1)-(1.5), expanded to become eigenfunctions of $A$, are complete in $H$. Namely, let $\left\{\Phi_{n}=\left(\varphi_{n}(x), T_{0}\left(\varphi_{n}\right), T_{1}\left(\varphi_{n}\right)\right) ; n \in\right.$ $\mathbb{N}\}$ be a maximum set of orthogonal eigenfunctions of $A$, then for all $F \in H, F=\sum_{n=1}^{\infty}\langle F$, $\left.\Phi_{n}\right\rangle \Phi_{n}$, where $\left\{\varphi_{n}(x) ; n \in \mathbb{N}\right\}$ are eigenfunctions of Sturm-Liouville problem (1.1)-(1.5).

Remark 4.1 When $\delta_{1}=\delta_{2}=1$, the considered problem turns into continuous case.

Remark 4.2 When $f(\lambda)=\cot \alpha$, the boundary condition (1.2) degenerates to the left boundary condition in $[21,22]$.

Remark 4.3 When $a=0$ or $c=0$, we need to redefine a new Hilbert space and a new inner product, similar results hold.

\section{Conclusion}

This paper gives a detailed characterization of spectral properties of certain kind of Sturm-Liouville problems with intermediate discontinuous point. The novelty lies in that the Sturm-Liouville problems we considered here involve both singular endpoints and an intermediate discontinuous point. By a newly defined Hilbert space and fundamental solutions of the equations, we give the spectrum of these equations.

Funding

This research was supported by the NNSF of China (Nos. 11271225 and 11671227) and NSF of Shandong Province (ZR2017MA042)

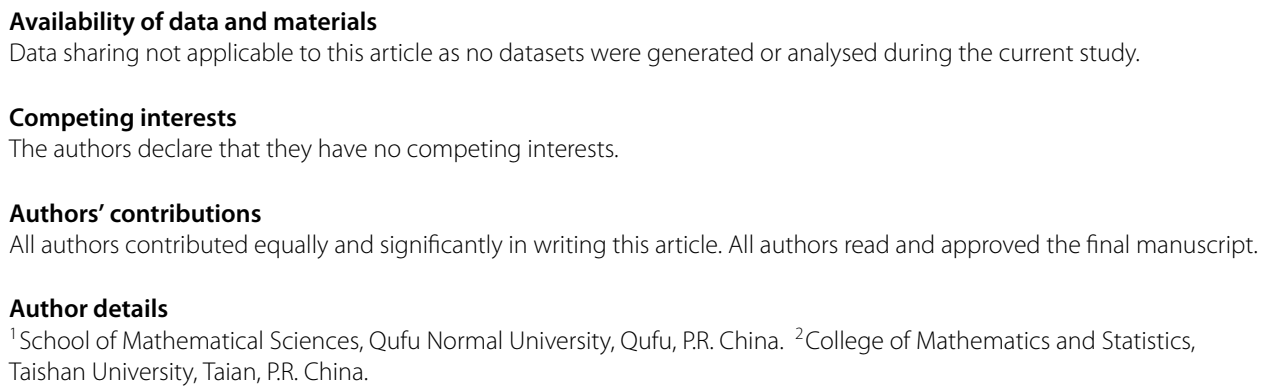

\section{Publisher's Note}

Springer Nature remains neutral with regard to jurisdictional claims in published maps and institutional affiliations. 


\section{References}

1. Collatz, L.: Eigenwertaufgaben mit technischen Anwendungen. Akad. Verlagsgesellschaft Geest Portig, Leipzig (1963)

2. Tretter, C.: Nonselfadjoint spectral problems for linear pencils $N-\lambda P$ of ordinary differential operators with $\lambda$-linear boundary conditions: completeness results. Integral Equ. Oper. Theory 26, 222-248 (1996)

3. Fulton, C.T.: Two-point boundary value problems with eigenvalue parameter contained in the boundary conditions. Proc. R. Soc. Edinb. A 77, 293-308 (1977)

4. Walter, J.: Regular eigenvalue problems with eigenvalue parameter in the boundary condition. Math. Z. 133, 301-312 (1973)

5. Friedman, B.: Principles and Techniques of Applied Mathematics. Wiley, New York (1956)

6. Freiling, G., Yurko, V.A.: Inverse problems for Sturm-Liouville equations with boundary conditions polynomially dependent on the spectral parameter. Inverse Probl. 26, 055003 (2010)

7. Qi, J., Zheng, Z., Sun, H.: Classification of Sturm-Liouville differential equations with complex coefficients and operator realizations. Proc. R. Soc. A 467, 1835-1850 (2011)

8. Zheng, Z., Ma, Y.: Dependence of eigenvalues of $2 m$ th-order spectral problems. Bound. Value Probl. 2017, 126 (2017). https://doi.org/10.1186/s13661-017-0857-y

9. Zheng, Z., Wang, X., Han, H.: Oscillation criteria for forced second order differential equations with mixed nonlinearities. Appl. Math. Lett. 22, 1096-1101 (2009)

10. Aydemir, K., Mukhtarov, O.S.: Variational principles for spectral analysis of one Sturm-Liouville problem with transmission conditions. Adv. Differ. Equ. 2016, 76 (2016)

11. Allahverdiev, B.P., Bairamov, E., Uğurlu, E.: Eigenparameter dependent Sturm-Liouville problems in boundary conditions with transmission conditions. J. Math. Anal. Appl. 401, 388-396 (2013)

12. Akdoğan, Z., Demirci, M., Mukhtarov, O.S.: Sturm-Liouville problems with eigendependent boundary and transmission conditions. Acta Math. Sci. 25, 731-740 (2005)

13. Mukhtarov, O.S., Yakubov, S.: Problems for ordinary differential equations with transmission conditions. Appl. Anal. 81, 1033-1064 (2002)

14. Li, K., Sun, J., Hao, X., Bao, Q.: Spectral analysis for discontinuous non-self-adjoint singular Dirac operators with eigenparameter dependent boundary condition. J. Math. Anal. Appl. 453, 304-316 (2017)

15. Cai, J., Zheng, Z.: A singular Sturm-Liouville problem with limit circle endpoints and eigenparameter dependent boundary conditions. Discrete Dyn. Nat. Soc. 2017, Article ID 9673846 (2017)

16. Zhang, M., Wang, Y.: Dependence of eigenvalues of Sturm-Liouville problems with interface conditions. Appl. Math Comput. 265, 31-39 (2015)

17. Li, K., Sun, J., Hao, X.: Eigenvalues of regular fourth-order Sturm-Liouville problems with transmission conditions. Math. Methods Appl. Sci. 40, 3538-3551 (2017)

18. Yang, Q., Wang, W.: Asymptotic behavior of a differential operator with discontinuities at two points. Math. Methods Appl. Sci. 34, 373-383 (2010)

19. Wang, A.: Research on Weidmann conjecture and differential operators with transmission conditions. PhD thesis, Inner Mongolia University (2006) (in Chinese)

20. Kadakal, M., Mukhtarov, O.S.: Sturm-Liouville problems with discontinuities at two points. Comput. Math. Appl. 54, 1367-1379 (2007)

21. Binding, P.A., Browne, P.J., Watson, B.A.: Sturm-Liouville problems with boundary conditions rationally dependent on the eigenparameter, I. Proc. Edinb. Math. Soc. 45, 631-645 (2002)

22. Binding, P.A., Browne, P.J., Watson, B.A.: Sturm-Liouville problems with boundary conditions rationally dependent on the eigenparameter, II. J. Comput. Appl. Math. 148, 147-168 (2002)

23. Cao, Z:: Ordinary Differential Operator, Shanghai (1986) (in Chinese)

24. Naimark, M.A.: Linear Differential Operators. Ungar, New York (1968)

25. Titchmarsh, E.C.: Eigenfunctions Expansion Associated with Second Order Differential Equations, Part 1. Oxford University Press, London (1962)

26. Zettl, A.: Sturm-Liouville Theory. Math. Surveys Monogr., vol. 121. Am. Math. Soc., Providence (2005)

27. Olğar, H., Muhtarov, F.S.: The basis property of the system of weak eigenfunctions of a discontinuous Sturm-Liouville problem. Mediterr. J. Math. 14, 114 (2017)

28. Mukhtarov, O.S., Olğar, H., Aydemir, K.: Resolvent operator and spectrum of new type boundary value problems. Filomat 29(7), 1671-1680 (2015)

29. Mukhtarov, O.S., Aydemir, K.: The eigenvalue problem with interaction conditions at one interior singular point. Filomat 31(17), 5411-5420 (2017)

30. Olğar, H., Mukhtarov, O.S., Aydemir, K.: Some properties of eigenvalues and generalized eigenvectors of one boundary-value problem. AIP Conf. Proc. 1759, 020060 (2016). https://doi.org/10.1063/1.4959674

31. Likov, A.V., Mikhailov, Y.A.: The Theory of Heat and Mass Transfer. Qosenerqoizdat, Moscow (1963) (Russian) 